\title{
Trauma Informed Developmentally Supportive Care
}

Kristy Fuller, OTR/L, CNT, CLC; Tara DeWolfe, DPT, CNT, CLC; Mary Coughlin BSN, RN, MS, NNP, RNC-E

Caring Essentials Collaborative, LLC Boston MA, USA

DOI: 10.14434/Do.v15ı1.33788

\section{Introduction}

Trauma informed developmentally supportive care creates a culture of care that focuses on the human experience. The concept of trauma informed care was co-opted from the field of behavioral and mental health in 2013 for application in the newborn intensive care unit (NICU). In essence, the concept realizes the pervasiveness of trauma in everyday life, recognizes signs and symptoms of trauma, responds to trauma through the adoption of evidence-informed best practices, and resists re-traumatization. Research across a wide range of scientific fields as diverse as neuroscience, molecular biology, epigenetics, developmental psychology, and psychoneuroimmunology verify intensive care hospitalization as a traumatic life event for babies, families, and clinicians. ${ }^{1,2}$ This experience is associated with significant morbidity and mortality across physiological, psychological, socioemotional, and spiritual domains. ${ }^{1-6}$ Most recently, the American Academy of Pediatrics (AAP) has issued a policy statement summarizing the urgency and requisite steps necessary to facilitate the integration of trauma informed principles into all pediatric points of care. ${ }^{3}$ "Trauma-informed care (TIC) in child health care operationalizes the biological evidence of toxic stress with the insights of attachment and resilience to enhance healthcare delivery to mitigate the effects of trauma". ${ }^{3}$

\section{What is trauma?}

According to the Substance Abuse and Mental Health Services Administration, trauma results from an event, a series of events, or a set of circumstances that is experienced by an individual as physically and/or emotionally harmful or life-threatening and has lasting adverse effects on the individual's functioning as well as their mental, physical, social, emotional, and/or spiritual well-being. ${ }^{7}$ Within the context of the NICU, infants experience trauma in big and small ways; from life-threatening illness to the day-to-day experiences of parental separation, fragmented sleep, a disruptive environment, painful experiences, stressful eating encounters, limited mobility, prolonged time in bed, and so much more. Maternal separation is the most significant trauma experienced by all newborn mammals and preterm and/or critically ill newborns are no exception to this reality. Morgan et al. discovered that separation of mother and baby at two days of age for one hour results in a $176 \%$ increase in autonomic activity and an $86 \%$ reduction in quiet sleep with sleep cycling almost abolished during the separation period. ${ }^{8}$ In the NICU, the experience of maternal separation becomes the foundation for cumulative toxic stress exposures that include unmanaged or underman- aged stress and pain, sleep fragmentation, susceptibility to inappropriate sensory stimuli from the physical and social environments, postural malalignment, and hazardous rituals and routines that do not honor the personhood of the baby. ${ }^{9}$ The trauma experienced in this setting, however, is not limited to the baby but encompasses the family's experience as well as the experience of the clinician who bears witness to the lived trauma of both baby and family.

\section{Why is trauma informed developmental care important?}

Adversity during childhood impacts the developmental trajectory of health and wellness across the lifespan. ${ }^{2}$ Individuals who survive childhood adversity are at greater risk for cardiovascular disease, metabolic syndromes, generalized anxiety disorders, depression, and other forms of psychopathology as well as central sensitizing syndromes that encompass fibromyalgia, migraines, and irritable bowel syndrome to name a few. ${ }^{10}$ In a cohort study of more than six million individuals with a birth history of varying degrees of prematurity there is a clear association of increased risk for premature adult death associated with non-communicable diseases; however, the most common cause of premature death in individuals born premature is accidents and suicide. ${ }^{11}$

In addition to the trauma endured by the hospitalized baby, the family experience of NICU hospitalization has been described as a traumatic event that shatters hopes, dreams, and expectations for parenthood. Roque et al. report the significant impact the NICU experience has on the emotional and mental health of parents. ${ }^{12}$ Outcomes include an increased risk of depression, anxiety, and post-traumatic stress disorder for both mothers and fathers because of their baby's NICU hospitalization. ${ }^{12}$ Finally, research highlights the effect of bearing witness to tragedy and trauma on clinicians' physical, emotional, and spiritual health. Bearing witness is a "human-to-human way of being-relating" to the truth of another's experience. ${ }^{13}$ However, when clinicians are not aware of the dynamic nature of trauma and trauma exposure it may limit their ability to interact in meaningful and safe ways with patients and families while also disrupting their personal wholeness. ${ }^{14}$

\section{Why a trauma informed approach to care?}

Trauma informed developmental care invites individuals to move past procedure-driven routines, rituals, and practices; to shift from a dualistic perspective on healthcare to a wholistic perspective. A trauma informed approach enables clinicians to not only understand the biological implications of trauma, 


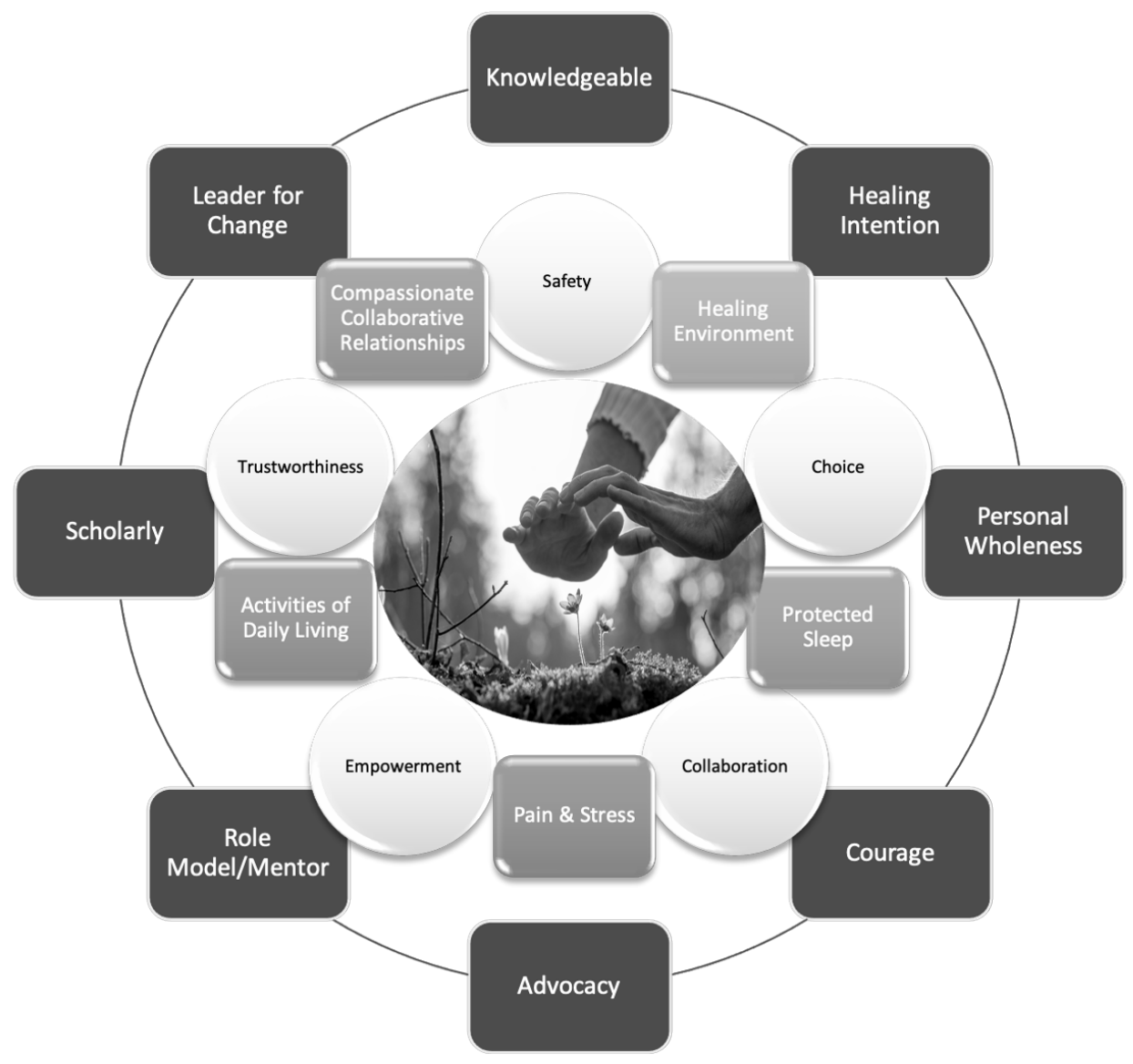

Endorsed by the NIDCAP Federation International, the National

Association of Neonatal Nurses, the Council of International Neonatal Nurses and the National Association of Perinatal Social Workers, the program combines online and virtual/live education to support clinicians in developing competence across the eight attributes of the Trauma Informed Professional. (Learn more: https://www. caringessentials.net/).

but to then take the necessary actions to buffer the trauma experience. Policies, protocols, and practices don't transform an experience, people do. Exploring trauma informed care begins with getting in touch with one's own story. It is through personal self-discovery that one can connect authentically with others and begin the paradigm shift.

"In the process of creating transformation we ourselves are transformed" - Mary Coughlin

\section{How to create transformation?}

Personal transformation is the key to cultural transformation. In the words of Carl Jung, "Your vision will become clear only when you can look into your own heart. Who looks outside, dreams, who looks inside, awakens". (Carl Jung, Letters Vol 1 1906-1950, p33, Princeton University Press, 1973). Becoming a Trauma Informed Professional (TIP) is a journey of self-discovery and personal growth that awakens the individual to their potentiality as a leader for change and a healer of hurts that too often go unnoticed in the highly technological world of newborn intensive care. Eight attributes of the Trauma Informed Professional have been established by an international, interdisciplinary faculty board of neonatal experts. These attributes are:
- Knowledgeable: Understanding core knowledge regarding the science underpinning early life adversity and the biologic sequelae.

- Healing intention: Fostering intention and presence, a cornerstone of trauma informed care, and based upon unitary caring science theory.

- Personal wholeness: Developing habits to support physical, psychological, social, spiritual, and existential well-being.

- Courage: Taking courageous action to effect change, increase self-actualization, and reduce moral distress by respectfully challenging the status quo.

- Advocacy: Showing up for self and others by demonstrating respectful influence and inspiring excellence.

- Role model and mentor: Integrating the qualities of humility and empathy as a role model and mentor to inspire others to greater success.

- Scholarly: Pursuing scholarship to consider all ways of sharing information.

- Leader for change: Cultivating a respectful approach to leadership as a Trauma Informed Professional is transformational. 


\section{Collaborative Caring}

The aligned missions of Caring Essentials Collaborative and the NIDCAP Federation International have led to collaborative teamwork aimed at transforming the experience of care for babies, families, and clinicians in the NICU and beyond. The complementary nature of NIDCAP Certification and the Trauma Informed Professional Certificate Program serves to advance the competencies of the bedside clinician empowering these individuals to become confident leaders for change. NIDCAP observers bear witness to the tiniest expressions of suffering, beyond what others can see. This depth of awareness takes a toll on the observer. Becoming a trauma informed NIDCAP professional mitigates this impact on self and others. Integrating trauma informed developmentally supportive care in the NICU and beyond changes not only individual lives but society at large. Together, we can design the future of truly compassionate healthcare.

\section{References}

1. Coughlin M. Transformative Nursing in the NICU: Trauma-informed Age-appropriate Care. 2nd ed. New York: Springer Publishing Company; 2021

2. Shonkoff JP, Garner AS, Committee On Psychosocial Aspects Of Child And Family Health, Committee On Early Childhood, Adoption, And Dependent Care, Section On Developmental And Behavioral Pediatrics. The lifelong effects of early childhood adversity and toxic stress. Pediatrics. 2012 Jan; 129(1): e232-e246. doi:10.1542/peds.2011-2663.

3. Duffee J, Szilagyi M, Forkey H, Kelly ET, Council On Community Pediatrics, Council On Foster Care, Adoption, And Kinship Care, Council On Child Abuse And Neglect, Committee On Psychosocial Aspects Of Child And Family Health. Trauma-informed care in child health systems. Pediatrics. 2021 Aug;148(2):e2021052579. DOI: https://doi. org/10.1542/peds.2021-052579.

4. Forkey H, Szilagyi M., Kelly ET, Duffee J, Council On Foster Care, Adoption, And Kinship Care, Council On Community Pediatrics, Council On Child Abuse And Neglect, Committee On Psychosocial Aspects Of Child And Family Health. Trauma-informed care. Pediatrics. 2021 Aug;148(2):e2021052580; DOI: https://doi.org/10.1542/peds.2021052580 .

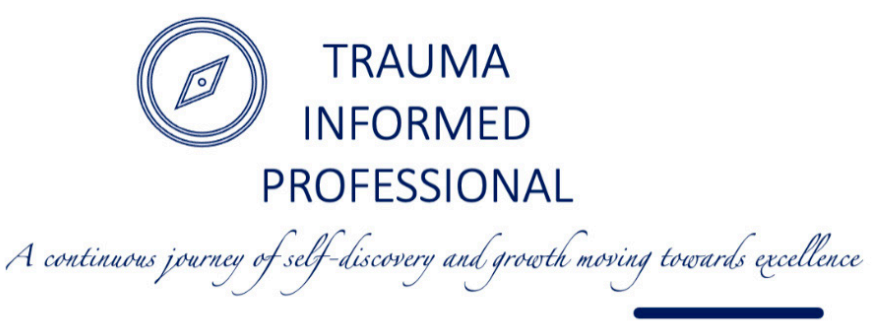

5. Montirosso R, Tronick E, Borgatti R. Promoting neuroprotective care in neonatal intensive care units and preterm infant development: Insights from the neonatal adequate care for quality-of-life study. Child Development Perspectives. 2017 Nov;11(1):9-15. https:// doi.org/10.1111/cdep.12208.

6. Sanders MR, Hall SL. Trauma-informed care in the newborn intensive care unit: promoting safety, security, and connectedness. Journal of Perinatology. 2018 Jan;38(1):3-10. doi: 10.1038/jp.2017.124.

7. Substance Abuse and Mental Health Services Administration. SAMHSA's Concept of Trauma and Guidance for a Trauma-Informed Approach. HHS Publication No. (SMA) 14-4884. Rockville, MD: Substance Abuse and Mental Health Services Administration, 2014.

8. Morgan B E, Horn AR, Bergman N J. Should neonates sleep alone? Biol Psychiatry. 2011 Nov 1;70(9):817-825. https://doi.org/10.1016/j. biopsych.2011.06.018.

9. Weber A, Harrison TM. Reducing toxic stress in the NICU to improve infant outcomes. Nurs Outlook. 2019 Mar-Apr;67(2):169-189. https://doi.org/10.1016/j.outlook.2018.11.002

10. Crump C. An overview of adult health outcomes after preterm birth. Early Hum Dev 2020 Nov;150:105187. doi: 10.1016/j.earlhumdev.2020.105187.

11. Risnes K, Bilsteen JF, Brown P, Pulakka A, Anderson A-MN, Opdahl, S., et al. Mortality among young adults born preterm and early term in 4 Nordic nations. JAMA Netw Open 2021 Jan 4;4(1):e2032779. doi: 10.1001/jamanetworkopen.2020.32779.

12. Roque ATF, Lasiuk GC, Radunz V, Hegadoren, K. Scoping review of the mental health of parents of infants in the NICU. J Obstet Gynecol Neonatal Nurs. 2017 JulAug;46(4):576-587. doi: 10.1016/j.jogn.2017.02.005.

13. Naef R. Bearing witness: a moral way of engaging in the nurse-person relationship. Nurs Philos. 2006 Jul;7(3):146-156. DOI: 10.1111/j.1466- 769X.2006.00271.x

14. Missouridou E. Secondary posttraumatic stress and nurses' emotional responses to patient's trauma. J Trauma Nurs. 2017 Mar/Apr;24(2):110-115. DOI: 10.1097/ JTN.0000000000000274.

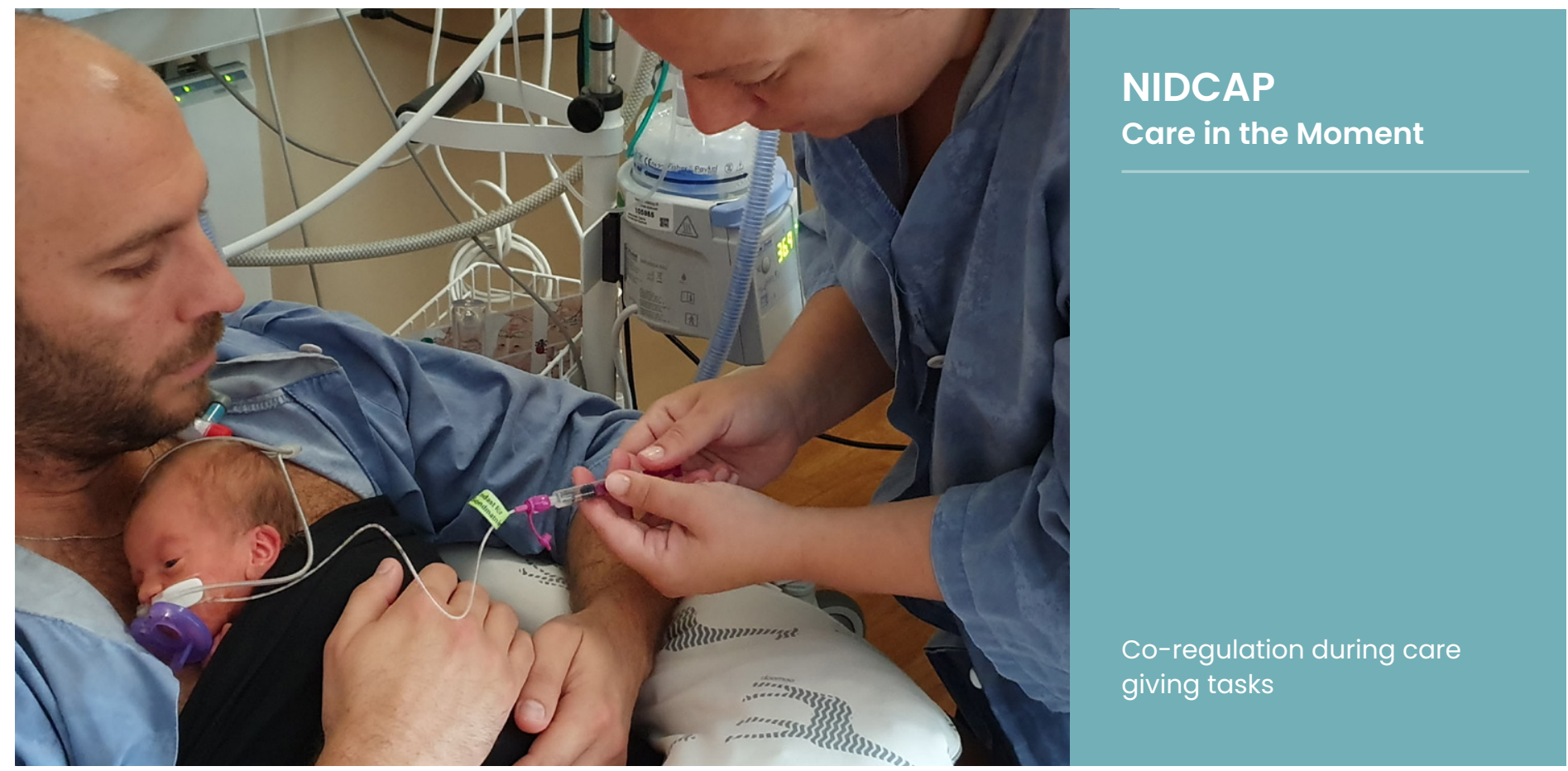

